\title{
On the Behavior of One Finite Automaton in a Fuzzy Environment
}

\author{
Tariel Khvedelidze ${ }^{1}$, Irma Aslanishvili ${ }^{2}$ \\ \{tariel.khvedelidze@tsu.ge ${ }^{1}$, irma.aslanishvili@tsu.ge ${ }^{2}$ \} \\ Faculty of Exact and natural Sciences, Department of Computer Sciences, \\ Iv. Javakhishvili Tbilisi State University, Tbilisi, Georgia
}

\begin{abstract}
Considered by the behavior of the finite automaton in a fuzzy environment that punishes or encourages an automaton with some fixed membership functions that are independent of time. Using the properties of the generalized Markov chain it is shown that the finite automaton under consideration in a fuzzy environment learns and more often performs that the action the punishment for which is minimal.
\end{abstract}

Keywords: Expediency of behavior, fuzzy environment, generalized Markov chain, learning automaton, membership function.

\section{Introduction}

The methods of the theory of Markov chains and processes are widely used in the study of processes of different nature. As an example, one can point to a description of the behavior of finite automata in a stationary random environment, which punishes or encourages an automaton for performing an action with some fixed probabilities. It should be noted that the beginning of a systematic study of the questions of the behavior of finite automata in a stationary random environment - as a task of choice one of several actions with random reinforcement - is connected M. L. Tsetlin's. His belongs owns the well-known construction of a finite automaton with linear tactics, which forms an asymptotically optimal sequence in a stationary random environment [1]. However, for the first time, the a task of sequentially choosing one of two methods of action, each of which can lead to success or failure was considered in [2]. Then V. I. Krinsky, by the same rule to construct a finite automaton and studied its behavior in a stationary random environment [3]. The developed approach for studying the behavior of automata in a stationary random environment in $[1,3]$ was based on the study of the final (at time $t \rightarrow \infty$ ) probabilities of Markov chains describing the behavior of these automata in a stationary random environment. It was believed that the sequence of finite automata of V. I. Krinsky, unlike a finite automaton with linear tactics by M. L. Tsetlin's, is asymptotically optimal (with respect to the memory capacity of the automaton $n \rightarrow \infty$ ) in any stationary random environment. However, further research showed [see e.g. 4] that this automaton in a probabilistic environment unconditional optimal behavior does not possess: its behavior in a random environment can turn out to be both optimal and anti-optimal. It should be noted that the study of the behavior of automata in a random environment is based on the classical apparatus of probability Markov chains, which has serious experimental support in statistics. For the analysis of systems with fuzzy information, such an experimental base is not 
yet available. Therefore, the results obtained in the this article can be considered as one example for the statistics fuzzy systems. An analysis of the behavior of automata in a fuzzy environment became possible after a certain generalization of the apparatus of Markov chains to non-probabilistic case [5]. Using this apparatus, in [6] the behavior of an automaton with linear tactics M. L. Tsetlin's was considered and it was shown that under certain additional conditions for environment parameters, the behavior of an automaton with linear tactics in a fuzzy environment is asymptotically optimal. In this paper considered the behavior of the finite automaton of V. I. Krinsky in a fuzzy environment, which punishes or encourages an automaton with some fuzzy membership functions. Using the properties of the generalized Markov chain it is shown that the finite automaton under consideration in a fuzzy environment learns and more often performs the action the punishment for which is minimal.

\section{The Behavior of The Finite Automaton V. I. Krinsky's in a Fuzzy Environment}

The finite automaton of V. I. Krinsky $D_{2 n, 2}$ has $2 n$ internal states $L^{(n)}=L_{1}^{(n)} \cup L_{2}^{(n)}, L_{1}^{(n)} \cap$ $L_{2}^{(n)}=\varnothing \quad$ and in the states of the region $L_{\alpha}^{(n)}, \alpha=1,2$ the automaton performs the action $f_{\alpha}$, $\alpha=1,2$. At encouragement, an automaton from any state of depth $x=j(j=1,2, \ldots, n)$ goes into the deepest state $x=n$ of the region $L_{\alpha}^{(n)}, \alpha=1,2$, and at punishment an automaton from any state of depth $x=j$ goes into an neighboring state depth $x=j-1, j=$ $2,3, \ldots, n$. A change in the actions of the automaton occurs from the state of depth $x=1$ : the automaton, being in the state of depth $x=1$ in the region $L_{\alpha}^{(n)}$ and receives a punishment, it goes into the state of the same depth $x=1$ of the region $L_{\beta}^{(n)}, \alpha, \beta=1,2, \alpha \neq \beta$. The graph of transitions between the states of the automaton $D_{2 n, 2}$ is shown in Figure 1.

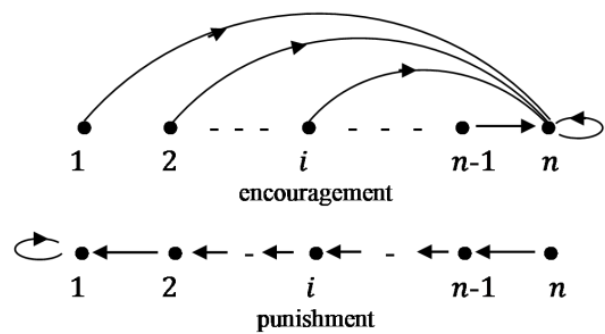

Fig.1. The graph of transitions between the states of the automaton with two actions $D_{2 n, 2}$ in the region

$$
L_{\alpha}^{(n)}, \alpha=1,2 .
$$

Let the automaton $D_{2 n, 2}$ be placed in a fuzzy environment and assume, as in [6], that $\mu_{j}^{(\alpha)}(t)$ means the function of belonging to states depth $x=j$ and appropriate the action $f_{\alpha}, \alpha=1,2$ at time $t=1,2, \ldots$. Assume that at each moment of time the automaton either receives a 
punishment with the membership function $\lambda_{\alpha}$, or a encouragement with the membership function $1-\lambda_{\alpha}, \quad \alpha=1,2$. We note that the quantities $\lambda_{\alpha}$ (hence $1-\lambda_{\alpha}$ ) $\alpha=1,2$ are unobservable quantities and they are time independent, i.e., the fuzzy environment is stationary. Note also, the transitions between the states of the automaton inside the region $L_{\alpha}^{(n)}$, $\alpha=1,2$ are identical and differ only in membership functions $\lambda_{\alpha}$ and $1-\lambda_{\alpha}, \alpha=1,2$ which means that the nature of the environment is not known in advance to the automaton. We will also assume that the "automaton-fuzzy environment" system is described by a generalized Markov chain [6], which is ergodic and, therefore, with time stationary membership quantities form in it. Then, by virtue of [5], we have that the "fuzzy flows" from $L_{1}^{(n)}$ in $L_{2}^{(n)}$ and are inversely balanced, i.e.

$$
\lambda_{1} \mu_{1}^{(1)}=\lambda_{2} \mu_{1}^{(2)} .
$$

Given the design of the automaton, relatively fuzzy flows inside the region $L_{1}^{(n)}$ we have that the fuzzy flow from the state of depth $x=j$ to the state of depth $x=j-1$ is equal to the sum of fuzzy flows from the state of depth $2,3, \ldots, j-1$. Thus have:

$$
\begin{gathered}
\lambda_{1} \mu_{2}^{(1)}=\left(1-\lambda_{1}\right) \mu_{1}^{(1)}, \\
\lambda_{1} \mu_{3}^{(1)}=\left(1-\lambda_{1}\right)\left(\mu_{1}^{(1)}+\mu_{2}^{(1)}\right), \\
\cdots \cdot \lambda^{i-1} \cdot \\
\lambda_{1} \mu_{i}^{(1)}=\left(1-\lambda_{1}\right) \sum_{k=1}^{1-1} \mu_{k}^{(1)}, \quad i=2,3, \ldots, n, \\
\cdot \cdot \cdot \cdot \cdot \\
\lambda_{1} \mu_{n}^{(1)}=\left(1-\lambda_{1}\right)\left(\mu_{1}^{(1)}+\mu_{2}^{(1)}+\ldots+\mu_{n-1}^{(1)}\right) .
\end{gathered}
$$

Similarly, for the region $L_{2}^{(n)}$ will have:

$$
\begin{gathered}
\lambda_{2} \mu_{2}^{(2)}=\left(1-\lambda_{2}\right) \mu_{1}^{(2)}, \\
\lambda_{2} \mu_{3}^{(2)}=\left(1-\lambda_{2}\right)\left(\mu_{1}^{(2)}+\mu_{2}^{(2)}\right), \\
\cdot \cdot \cdot \cdot \cdot \cdot \\
\lambda_{2} \mu_{i}^{(2)}=\left(1-\lambda_{2}\right) \sum_{k=1}^{i-1} \mu_{k}^{(2)}, \quad i=2,3, \ldots, n, \\
\cdot \cdot \cdot \cdot \cdot \\
\lambda_{2} \mu_{n}^{(2)}=\left(1-\lambda_{2}\right)\left(\mu_{1}^{(2)}+\mu_{2}^{(2)}+\ldots+\mu_{n-1}^{(2)}\right) .
\end{gathered}
$$

From (2) and (3) express all $\mu_{j}^{(1)}$ and $\mu_{j}^{(2)} \quad(j=2,3, \ldots, n)$ through $\mu_{1}^{(1)}$ and $\mu_{1}^{(2)}$ respectively. As a result, obtain 


$$
\begin{gathered}
\mu_{j}^{(1)}=\frac{1-\lambda_{1}}{\lambda_{1}{ }^{j-1}} \mu_{1}^{(1)}, \quad \mu_{j}^{(2)}=\frac{1-\lambda_{2}}{\lambda_{2}{ }^{j-1}} \mu_{1}^{(2)}, \\
j=2,3, \ldots, n .
\end{gathered}
$$

From (1)

and finally will have

$$
\mu_{1}^{(2)}=\frac{\lambda_{1}}{\lambda_{2}} \mu_{1}^{(1)}
$$

$$
\begin{gathered}
\mu_{j}^{(1)}=\frac{1-\lambda_{1}}{\lambda_{1}{ }^{j-1}} \mu_{1}^{(1)}, \\
\mu_{j}^{(2)}=\frac{\lambda_{1}}{\lambda_{2}} \frac{1-\lambda_{2}}{\lambda_{2}^{j-1}} \mu_{1}^{(1)}, \\
j=2,3, \ldots, n .
\end{gathered}
$$

We now define the fuzzy membership functions $M^{(\alpha)}$ of the state of the automaton to the region $L_{\alpha}^{(n)}, \alpha=1$, 2. In [7], it was shown that the final membership function $\theta$ coming from $n$ sources $\beta_{1}, \beta_{2}, \ldots, \beta_{n}$ is determined by the expression

$$
\theta\left(\beta_{1}, \beta_{2}, \ldots, \beta_{n}\right)=\sum_{i} \beta_{i}-\sum_{i \neq j} \beta_{i} \beta_{j}+\sum_{i \neq j \neq k} \beta_{i} \beta_{j} \beta_{k}-\ldots
$$

which can be rewritten in the following form

$$
\theta\left(\beta_{1}, \beta_{2}, \ldots, \beta_{n}\right)=1-\left(1-\beta_{1}\right)\left(1-\beta_{2}\right) . .\left(1-\beta_{n}\right)=1-\prod_{i=1}^{n}\left(1-\beta_{i}\right) .
$$

Then the fuzzy final membership function $M^{(1)}$ of the state of the automaton of the region $L_{1}^{(n)}$, in which the automaton performs the first action,

$$
M^{(1)}=1-\prod_{j=1}^{n}\left(1-\mu_{j}^{(1)}\right)=1-\left(1-\mu_{1}^{(1)}\right) \prod_{j=2}^{n}\left(1-\frac{1-\lambda_{1}}{\lambda_{1}^{j-1}} \mu_{1}^{(1)}\right) .
$$

Similarly, for the second action of the automaton, we obtain

$$
M^{(2)}=1-\prod_{j=1}^{n}\left(1-\mu_{j}^{(2)}\right)=1-\left(1-\mu_{1}^{(2)}\right) \prod_{j=2}^{n}\left(1-\frac{1-\lambda_{2}}{\lambda_{2}^{j-1}} \mu_{1}^{(2)}\right),
$$

which can be expressed in terms of $\mu_{1}^{(1)}$ in the following form

$$
M^{(2)}=1-\left(1-\frac{\lambda_{1}}{\lambda_{2}} \mu_{1}^{(1)}\right) \prod_{j=2}^{n}\left(1-\frac{\lambda_{1}}{\lambda_{2}} \frac{1-\lambda_{2}}{\lambda_{2}^{j-1}} \mu_{1}^{(1)}\right) .
$$


The value of the positive quantity $\mu_{1}^{(1)} \leq 1$ in the expressions $M^{(1)}$ and $M^{(2)}$ can be found from the obvious consideration $M^{(1)}+M^{(2)}=1$. We now analyze expressions (5) and (6). Suppose that the automaton's memory depth $\mathrm{n}$ is finite and the membership function for the punishment for the action $f_{1}$ is less than the membership function for the punishment for the action $f_{2}$, i.e. $\lambda_{1}<\lambda_{2}$ (therefore, the membership function for the encouragement for the action $f_{1}$ will be greater than the membership function for the encouragement for the action $f_{2}$, i.e. $1-\lambda_{1}>1-\lambda_{2}$ ). Then from (5) and (6) it is easy to establish that $M^{(1)}>M^{(2)}$. Now let $n \rightarrow \infty$. Then from (5) and (6) it is unambiguously impossible to establish which of the quantities $M^{(1)}$ and $M^{(2)}$ is greater. Indeed, taking into account the design of the automaton, one can make the following analysis: if at the initial instant of time the automaton is in the state of the region $L_{\alpha}^{(n)}, \alpha=1,2$, then at the first encouragement it can end up in the deepest state of one or another areas and from which it will be practically impossible to exit (therefore, change actions) in a finite number ofstep. Consequently, the behavior of the automaton can turn out to be both optimal and anti-optimal.

\section{Conclusion}

The results obtained show at functioning finite automaton V. I. Krinsky's in a fuzzy environment, be observed the fact of his learning and in a fuzzy environment its behavior is expedient: automaton in a fuzzy environment often performs the action for which the punishment is minimal. Regarding asymptotic optimality, we can say that V. I. Krinsky's finite automaton in a fuzzy environment not have obvious optimal behavior: its behavior in a fuzzy environment can turn out to be both optimal and anti-optimal. Thus, the finite automaton of V. I. Krinsky in a fuzzy environment behaves in the same way as in a probabilistic environment.

\section{References}

[1] Tsetlin M.L. On the behavior of finite automata in random environments. Automation and telemechanics, N10 (1961)

[2] Robbins H. A Sequential DecisionProblem with a Finite Memory. Proc.Of Nat. Acad. Of Science of USA, 42, 3 (1956)

[3] Krinsky V.I. Asymptotically optimal automaton with exponential rate of convergence. Biophysics 9, issue 4, pp. 67-71 (1964)

[4] Korolyuk V.S., Pletnev A.I., Eidelman S.D. Automata. Walks. Games. Successes of Mathematical Sciences. T. 43, issue 1 (259), pp. 87-122 (1988)

[5] Stefanuk V.L. Generalized Markov chains. Artificial Intelligence and Decision Making, N4, pp. 95$99(2011)$

[6] Stefanuk V.L.. Behavior of The Finite Automaton in a Fuzzy Environment: Theory and Applications. Artificial Intelligence and Decision Making, N3, pp. 54-61 (2014)

[7] Stefanuk V.L.. Do you trust the evidence? All-Union Conference on Artificial Intelligence. T. 1. M.: USSR Academy of Sciences, pp. 406- 410 (1988) 